\title{
The effect of a Pilates exercise programme on perceived functional disability and pain associated with non-specific chronic low back pain
}

\begin{abstract}
Non-specific low back pain (LBP) is a symptom of unknown cause affecting one third of the UK adult population, accounting for more than 15 million lost work days and an estimated annual cost of $£ 12.3$ billion. Pilates based exercise is becoming increasingly incorporated in to spinal rehabilitation, yet there is limited evidence on the efficacy of the technique. The aim of this study was to evaluate the effect of a 6-week Pilates based exercise programme on perceived functional disability, pain, local muscular endurance and lumbar range of motion in patients with non-specific chronic low back pain. Participants $(\mathrm{n}=13)$ with chronic non-specific LBP $(>12$-weeks) were recruited to a pre-post intervention trial. All participants were assigned to a 6-week control period. Subsequently, participants undertook one hour of a Pilates based exercise intervention twice per week for 6-weeks. Perceived functional disability and pain were assessed using the Oswestry functional disability questionnaire (ODQ) and visual analogue scale (VAS) respectively. Local muscular endurance (CE) and lumbar range of motion (LF) were assessed using the core endurance test and the fingertip to floor distance (FFD) respectively. Functional disability (ODQ), pain (VAS), local muscular endurance and lumbar flexion (FFD) improved after the intervention period $(\mathrm{P}<0.05)$. Improvements in perceived functional disability (ODQ) were associated with an increase in LF $(\mathrm{P}<0.05)$ but not VAS or CE $(\mathrm{P}>0.05)$. The results of this prepost intervention trial suggest that Pilates based exercise can be administered safely and is well tolerated by the majority of patients with non-specific chronic LBP.
\end{abstract}

Keywords: pilates, low back pain, exercise, therapy, functional disability, core stability, core endurance, lumbar flexion
Volume 2 Issue I - 2017

\author{
Llewellyn Helen, ${ }^{12}$ Konstantaki Maria, ${ }^{2}$ \\ Johnson Mark ${ }^{1,3}$ Francis Peter ${ }^{1}$ \\ 'Department of Musculoskeletal Health Research Group, School \\ of Clinical and Applied Sciences, Leeds Beckett University, \\ United Kingdom \\ ${ }^{2}$ Department of Applied Health \& Exercise Sciences, \\ Buckinghamshire New University, United Kingdom \\ ${ }^{3}$ Centre for Pain Research, School of Clinical and Applied \\ Sciences, Leeds Beckett University, United Kingdom
}

Correspondence: Helen Llewellyn, Department of Musculoskeletal Health Research Group, School of Clinical and Applied Sciences, Leeds Beckett University, United Kingdom, Tel 0II3 8I23845, Email helen.llewellyn@leedsbeckett.ac.uk

Received: November 23, 2016 | Published: March 17, 2017
Abbreviations: LBP, low back pain; TrA, transverse abdominis; IO, internal oblique; MF, multifidus; ODQ, oswestry functional disability questionnaire; VAS,visual analogue scale; LSN, lumbar spine neutral; FFD, fingertip to floor distance; CE, core endurance; LF, lumbar flexion

\section{Introduction}

Non-specific low back pain (LBP) is a symptom of unknown cause affecting one third of the UK adult population. ${ }^{1}$ In 2013 the UK Government ${ }^{2}$ reported that back pain was the leading cause of long-term sickness and accounted for more than 15 million lost work days with an estimated annual cost of $£ 12.3$ billion. ${ }^{3}$ However the therapeutic management of LBP varies widely and the effectiveness associated with many interventions has not been definitively demonstrated. ${ }^{4}$ Exercise has been reported as an effective strategy for the management of LBP. ${ }^{5}$ However the most suitable method of exercise in the treatment of LBP has been widely debated. ${ }^{6-10}$ Recently Pilates based exercises have been increasingly incorporated into physiotherapy rehabilitation programmes. ${ }^{11}$ The Pilates technique focuses on the principles of centring, postural alignment, co-ordination, concentration, breathing, precision and movement sequencing. ${ }^{12}$ These principles aim to allow dysfunctional movement to be isolated perfected then integrated back into functional movement ${ }^{13}$ and teach control of spinal movement during activities of daily living. ${ }^{14}$ The centring principle consists of an isometric contraction of the transverse Abdominis (TrA) and the internal oblique (IO) muscles which contributes to increased antagonist co activation of the deep lumbar muscles viz. multifidus (MF) ${ }^{15}$ Several authors have suggested that Pilates based exercise incorporates lumbar stabilisation training within dynamic and functional movement. ${ }^{13,16,17}$ Lumbar stabilisation in a functional context may be key to improving patient self-efficacy and reducing fear avoidance. ${ }^{18}$

Chronic LBP can impact on functional activities of daily living. The Oswestry Disability Questionnaire (ODQ) was designed to assess the impact of back pain on activities of daily living and has been shown to be a valid and responsive measure of functional disability. ${ }^{19,20}$ Scores from the ODQ are expressed as percentages and interpreted as, $<20 \%$ minimal disability, $21-40 \%$ moderate disability, $41-60 \%$ severe disability, $61-80 \%$ crippling back pain, $81-100 \%$ bed bound or patients were exaggerating symptoms. A ten percent change in ODQ score is considered the minimum acceptable change to be clinically meaningful. ${ }^{21}$ Interlinked with functional disability is the patient's perception of pain. Pain severity and relief is commonly evaluated using the Visual Analogue Scale (VAS) ${ }^{22}$ The VAS consists of a $100 \mathrm{~mm}$ straight line anchored by 0 (no pain) and 10 (worst pain). The recommended cut offs for the VAS are no pain $(0-4 \mathrm{~mm})$, mild pain $(5-44 \mathrm{~mm})$, moderate pain $(45-74 \mathrm{~mm})$ and severe pain (75-100mm). ${ }^{23}$ The VAS is regarded as a valid tool to measure pain severity in chronic pain patients and a reduction of $13 \mathrm{~mm}$ represents the minimum change in pain to be clinically significant. ${ }^{24}$ 
Activities of daily living such as bending down to lift an object from the floor require a combination of strength and range of motion. Impairments in core endurance have been associated with LBP and lower extremity injuries in athletes. ${ }^{25}$ The core endurance test is a measure of trunk control and can be used to assess the fatigability of the trunk muscles ${ }^{26}$ with moderate to high intra-ratter reliability $(\mathrm{ICC}=0.66-0.96) .{ }^{25}$ Akaha et al. ${ }^{27}$ reported that deficiencies in trunk flexibility closely reflected self-reported disability in activities of daily living and the fingertip to floor distance (FFD) test measures trunk flexibility and can be used to study lumbar impairment. Pilates based exercise has been suggested to demonstrate positive effects for improved general function and pain reduction with some evidence to support improvements in flexibility and enhanced muscle endurance ${ }^{28}$ and as such may offer an alternative cost effective method in the management of LBP. ${ }^{29}$ Increasingly therapists in Australia and the United States are using Pilates as an intervention in the management of chronic $\mathrm{LBP}^{12}$ despite minimal scientific evidence to support the technique..$^{11,14}$ Therefore the aim of this study was to evaluate the effect of a 6-week Pilates based exercise programme on perceived functional disability, pain, local muscular endurance and lumbar range of motion associated with non-specific chronic low back pain.

\section{Methods}

\section{Participants and procedures}

Fifteen physically active (defined by the UK Government ${ }^{30}$ as 150 minutes of moderate intensity activity per week) participants (Female $n=10$, age $57.4 \pm 6.6$ years, Male $n=5$, age $50.4 \pm 9.9$ years) with nonspecific LBP (>12 weeks) were recruited to this study. Exclusion criteria included pregnancy, manual treatment within the previous 3 months, positive straight leg raise test, prior spinal surgery, known lumbar disc hernia, severe osteoporosis and diagnosed inflammatory or malignant disease. ${ }^{31}$ Two participants were excluded from the study due to receiving previous spinal surgery. The study was designed as prospective pre-post intervention trial as it is deemed unethical to deny any participant with LBP access to treatment. ${ }^{32}$ Participants undertook a 6-week control period in which they continued their daily and physical activities as normal. Subsequently participants commenced one hour of a supervised (1:4 ratio) Pilates based exercise intervention twice per week for 6-weeks. After a complete explanation of the procedures benefits and risks associated with the study participants were invited to participate and to provide written informed consent. All procedures were performed in accordance with the most recent version of the Declaration of Helsinki and approved by faculty research ethics committee of Buckinghamshire New University.

\section{Perceived functional disability and pain}

Perceived functional disability and pain were assessed pre and post control and intervention periods using the ODQ and VAS respectively. Participants were requested to complete the applicable sections of the ODQ and their percentage of functional disability was calculated on the number of questions completed. Levels of perceived pain in the previous 24 -hour period were established by participants marking a vertical mark through the $100 \mathrm{~mm}$ horizontal VAS the distance was measured and recorded in millimetres $(\mathrm{mm})$.

\section{Local muscular endurance and lumbar range of motion}

Local muscular endurance was established by conducting a core endurance test in accordance with protocols established by Mc Gill. ${ }^{33}$ Participants were instructed to hold a static position for a long as possible the test was terminated when the subject could no longer sustain the position and the time recorded in seconds (s). Lumbar flexion range of motion was assessed using fingertip to floor distance (FFD). Participants were instructed stand with feet hip width a part arms held straight in front with hands together then to slowly bend forward in an attempt to touch the floor. The distance between the tip of the middle finger and the floor was measured and recorded in centimetres $(\mathrm{cm})$.

\section{Pilates based exercise}

Participants completed one hour of a supervised (1:4 ratio) Pilates exercise twice per week for 6-weeks. Each session included a 10-minute warm-up concentrating on movements of the trunk musculature. Participants were taught to find lumbar spine neutral (LSN) as the intermediate position between retroversion and anteversion. Activation and control of the TrA and MF muscles was established with expiration during diaphragmatic breathing in different positions (standing, sitting, supine, prone and four-point kneeling) based on the Pilates technique. The exercise intervention Figure 1 included the following Pilates movements, spine stretch forward, spine twist, single leg stretch, rolling, the hundred, diamond press, side kick and shoulder bridge. Exercise intensity and duration was progressed in accordance with the $\mathrm{ACSM}^{34}$ guidelines for chronic low back pain.

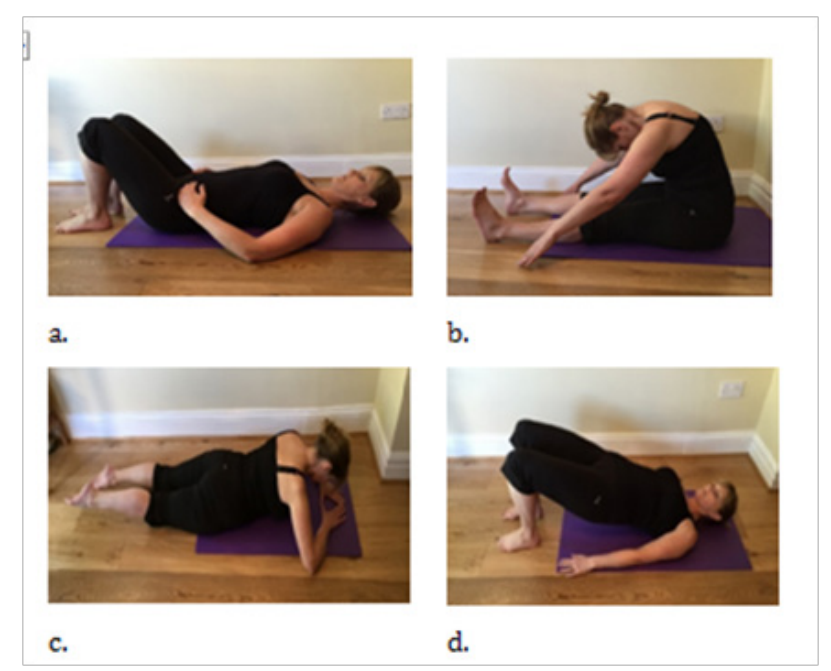

Figure I Pilates Intervention (A) LSN, (B) spine stretch forward, (C) diamond press, (D) shoulder bridge.

\section{Statistical analysis}

To assess normality of the data distribution a Shapiro-Wilk test was used. Descriptive statistics are reported as mean \pm standard deviation and median (Interquartile range). A paired samples $t$-test was used to analyze differences for both the control and intervention periods from baseline to 6-weeks for core endurance (CE) and lumbar flexion (LF). Where data were not normally distributed a non-parametric related-samples Wilcoxon signed rank test was performed to analyze perceived functional disability (ODQ) and pain (VAS). A Spearman's rank-order correlation was used to investigate any association between percentage change in LF, VAS, CE and ODQ after the 6-week Pilates intervention. Analyses of data were performed using the statistical 
software SPSS 22 for Windows (SPSS Inc, Chicago IL, USA). A statistical significance value of $\mathrm{P}<0.05$ was accepted.

\section{Results}

Participant recruitment, exclusion, participation and withdrawal are presented in Figure 2. Twelve participants (Female $n=7$, age $55.7 \pm 6.6$ years, Male $n=5$, age $50.4 \pm 9.9$ years) completed the study and their data was analysed. One participant performed a bout of eccentric exercise (unaccustomed) 24 hours prior to the end of the intervention which led to a $550 \%$ increase in pain over and above the range for all participants $(-74 \%$ to $+67 \%)$. This data was removed from further analysis. There was no difference in outcome measures after the 6-week control period Table 1. Functional disability (ODQ), pain (VAS), core endurance and lumbar flexion improved after the intervention period $(\mathrm{P}<0.05)$ Table 2. Mean and individual relative percentage changes in functional disability (ODQ), pain (VAS), core endurance and lumbar flexion after the 6-week Pilates intervention are displayed in Figure 3. Relative percentage change in perception of functional disability had a moderate $(r=0.645, \mathrm{P}=0.032)$ association with improvements in lumbar flexion Table 3. Changes in perceived pain and core endurance were not associated with changes in perception of functional disability $(\mathrm{P}>0.05)$.

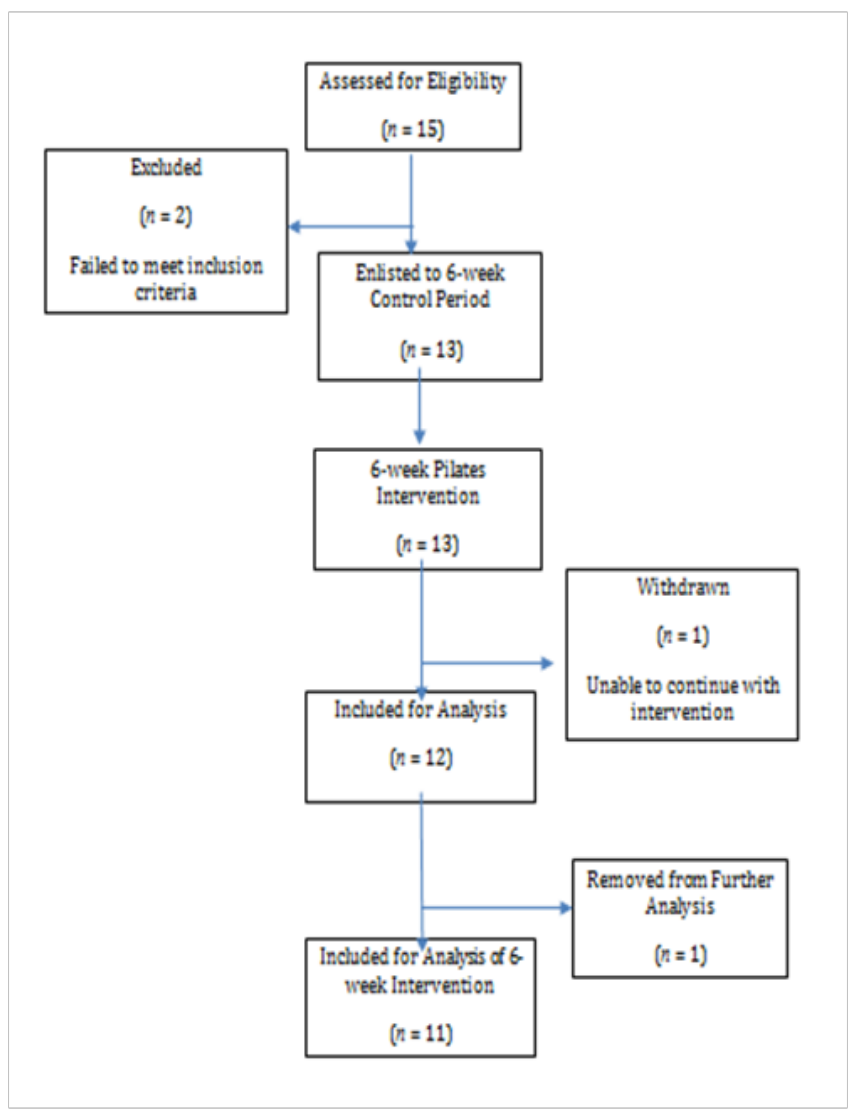

Figure 2 Study design.
Table I Outcome measures at baseline and after the 6-week control period

\begin{tabular}{llll}
\hline & Baseline & 6-week & P-value \\
\hline ODQ score (\%) & $17.7(9.5)$ & $17.7(9.5)$ & 0.317 \\
Range & $15.5-44.4$ & $15.5-48.8$ & \\
VAS (mm) & $35.5 \pm 17.5$ & $36.5 \pm 18.6$ & 0.307 \\
Range & $10-63$ & $10-70$ & \\
CE (s) & $11.9 \pm 4.6$ & $12.1 \pm 4.8$ & 0.137 \\
Range & $5.4-22.2$ & $5.4-22.5$ & \\
LF (cm) & $25.0 \pm 14.1$ & $25.08 \pm 14.0$ & 0.821 \\
Range & $4.0-50.0$ & $4.0-50.00$ & \\
\hline
\end{tabular}

Table 2 Changes in pain and function after a 6-week Pilate's intervention

\begin{tabular}{lllll}
\hline & Baseline & $\Delta$ 6-week & $\mathbf{\% \Delta}$ 6-week & P-value \\
\hline ODQ (\%) & $17.7(11.1)$ & $7.4 \pm 7.0$ & $33.6 \pm 28.2$ & 0.008 \\
VAS (mm) & $38.9 \pm 17.4$ & $15.1 \pm 18.3$ & $40.2 \pm 46.3$ & 0.036 \\
CE (s) & $11.2 \pm 3.7$ & $4.5 \pm 5.4$ & $57.9 \pm 70.8$ & 0.019 \\
LF (cm) & $26.5 \pm 13.8$ & $12(13)$ & $54.2 \pm 27.3$ & 0.003 \\
\hline
\end{tabular}

Table 3 Spearman Rank-Order Coefficient ( $r$ s) between percentage change in lumbar flexion (LF), perceived pain (VAS) and core endurance (CE) on functional disability (ODQ) after a 6-week Pilate's intervention

\begin{tabular}{llll}
\hline & LF & VAS & CE \\
\hline ODQ & $0.645, \mathrm{P}=0.032$ & $0.362, \mathrm{P}=0.275$ & $0.247, \mathrm{P}=0.465$ \\
\hline
\end{tabular}

\section{Discussion}

The results of this pre-post intervention trial suggest that Pilates based exercise can be administered safely and is well tolerated by the majority (11/12) of patients with non-specific chronic LBP. Furthermore mean absolute and relative changes indicated that it may have the potential to promote a clinically meaningful change in perceived functional disability and pain in this patient group. These mean changes are underpinned by $75 \%$ of the sample having a reduction $(>13 \mathrm{~mm})$ in pain post-intervention. Reductions in ODQ and VAS scores followed a similar trend for the majority of participants but it is not known if the reduction in perceived pain led to a reduction in functional disability. Improvements in LF and CE post intervention were observed for $92 \%$ and $75 \%$ of the sample respectively. Moreover increased LF was moderately associated with a reduction of perceived functional disability.

The results of the present investigation are in accordance with Taylor et al..$^{35}$ and Natour et al..$^{29}$ who observed improvements in pain and self-reported disability after 6 and 12-week Pilate's interventions respectively. Importantly our study observed mean decreases of 33\% in the ODQ and $15 \mathrm{~mm}$ in VAS scores which represents a clinically meaningful change. ${ }^{21,24}$ Both Taylor et al. ${ }^{35}$ and Natour et al. ${ }^{29}$ employed the VAS to measure pain and observed similar decreases of $19 \mathrm{~mm}$ and $15.9 \mathrm{~mm}$. However in contrast to our study Natour et al. ${ }^{29}$ measured functional disability using the Roland-Morris Disability Questionnaire which unlike the ODQ requires a response to all questions regardless of patient relevance. We cannot infer causation using the current study design nor did we investigate mechanisms of action associated with 
Pilates exercise although the improvements in functional disability may be suggestive of muscle recruitment (TrA, MF) which enhance static and dynamic posture. ${ }^{13}$ In a review of exercise interventions for LBP Searle et al. ${ }^{36}$ reported that interventions with an emphasis on isometric contractions of TrA contributed to lumbo-pelvic stability when stability was challenged. Furthermore Rio et al. ${ }^{37}$ observed that isometric contractions of the quadriceps femoris induced greater immediate analgesia in patella tendonopathy patients compared to concentric eccentric contractions. This has been suggested to increase the ability to load or perform in the post contraction analgesic phase..$^{28}$ However it is unclear whether the benefits of exercise to reduce perceived pain associated with chronic LBP are as a consequence of muscle loading or movement repetition. ${ }^{3} 8$

Improvements in the perception of functional disability and pain as a result of the Pilates exercise may contribute to improvements in core muscle endurance and range of motion. Richmond ${ }^{39}$ suggested exercise that transcends into integrated movement may improve resistance to fatigue of muscles associated with maintaining balance after a postural perturbation. CE improved post-intervention which is in accordance with the work of Natour et al. ${ }^{29}$ who purported that Pilates improved core strength because of higher levels of TrA contraction. In addition Kamioka et $a .^{28}$ reported that there was strong evidence that in a healthy population Pilates exercise improved flexibility and enhanced muscular endurance in the short term. While our study did not specifically measure TrA contraction Searle et al. [36] noted that isolated training of $\operatorname{TrA}$ led to an earlier onset of $\operatorname{Tr} \mathrm{A}$ activation which reflected a more constant activation pattern that is observed in healthy individuals. The increase in CE found in our study could not be attributed solely to TrA and MF contraction as the method of testing recruited both global and local musculature which was not specifically trained in the intervention. This may explain the varied response to changes in $\mathrm{CE}$ found in our study. Improvement in LF has a moderate association with a reduction in scores on the ODQ. This is in agreement with the hypothesis that improved perception of total lumbar flexion through FFD may reflect self-reported disability in activities of daily living. ${ }^{27}$ However this association must be interpreted cognisant that LF is measured using an objective test, unlike the OQD which relies on patient perception and interpretation of functional disability. The observed improvements in LF could be due to the exercises that were included in our study. Kumar et al. ${ }^{40}$ suggested exercises that created a stretch in the lumbar muscles, such as the spine stretch forward, rolling and spine twist performed in our study, could restore mobility which had been disturbed in chronic LBP. In contrast to our study Natour et al. ${ }^{29}$ did not find any significant improvements in LF. This may have been due to the disparity in testing procedures used to measure LF as Natour et al. ${ }^{29}$ used a sit and reach test which may not have sufficiently represented lumbar spine flexibility and could be influenced by hamstring flexibility. ${ }^{41}$

The current study established the short-term effects of the Pilates exercise on perceived functional disability and pain and objectively measured LF and CE. However the long-term efficacy and maintenance of improvements could not be established and this should be considered for future research.

\section{Conclusion}

The present study has provided sufficient trends to justify the use of a Pilates programme for the reduction of perceived functional disability and pain. In addition the Pilates exercise protocol improved core muscle endurance and lumbar flexibility. While it is acknowledged that further investigation is required into the proposed effects of the technique a Pilates based exercise intervention could offer a costeffective alternative to traditional manual therapy interventions for non-specific chronic LBP.

\section{Acknowledgements}

The author would like to thank Bannatyne's Health Club in Durham and Chester le Street for participant recruitment and the use of their facilities during this study.

\section{Conflict of interest}

The author declares no conflict of interest.

\section{References}

1. NICE. Low Back Pain: Early Management of Persistent Non-specific Low Back Pain. London, UK: Royal College of General Practitioners; 2009.

2. NHS. Back pain at work; 2015.

3. Whitehurst DG, Stirling B, Lewis M, et al. Exploring the cost-utility of stratified primary care management for low back pain compared with current best practice within risk-defined subgroups. Ann Rheum Dis. 2012;71(11):1796-1802.

4. Tulder VM, Malmivaara A, Esmail R, et al. Exercise therapy for low back pain: a systematic review within the framework of the Cochrane collaboration back review group. Spine. 2000;25(21):2784-2796.

5. Rainville J, Hartigan C, Martinez E, et al. Exercise as a treatment for chronic low back pain. Spine J. 2004;4(1):106-115.

6. Descarreaux M, Normand MC, Laurencelle L, et al. Evaluation of a specific home exercise program for low back pain. J Manipulative Physiol Ther. 2002;25(8):497-503.

7. Hayden JA, Tulder VMW, Tomlinson G. Systematic review: Strategies for using exercise therapy to improve outcomes in chronic low back pain. Ann Intern Med. 2005;142(9):776-785.

8. Moffet JK, Torgenson D, Syer BS, et al. Randomised controlled trial of exercise for low back pain: clinical outcomes, costs and preferences. BMJ. 1999;319(7205):279-283.

9. Koumantakis GA, Watson PJ, Oldham JA. Trunk muscle stabilization training plus general exercise versus general exercise only: randomized controlled trial of patients with recurrent low back pain. Phys Ther. 2005;85(3):209-225.

10. Richardson C, Jull G, Hodges P, et al. Therapeutic Exercise for Spinal Segmental Stabilization in Low Back Pain. London, England: Churchill Livingstone; 2002.

11. Miyamoto GC, Costa LO, Galvanin T, et al. The efficacy of the addition of the Pilates method over a minimal intervention in the treatment of chronic nonspecific low back pain: a study protocol of a randomized controlled trial. J Chiropr Med. 2011;10(4):248-254.

12. Massey P. Pilates in Sport-A tool for injury prevention and injury treatment. Sport EX Medicine. 2005;23:9-15.

13. Anderson BD, Spector A. Introduction to Pilates-based rehabilitation. Orthopaedic Physical Therapy Clinics of North America. 2000;9(3):395410 .

14. Dolan AM, Hutchinson MJ, Fraser RD. The Pilates based exercise programme in the management of low back pain. The Journal of Bone and Joint Surgery (Br). 2001;83(S1):82. 
15. Rossi DM, Morcelli MH, Marques NR, et al. Antagonist coactivation of trunk stabilizer muscles during Pilates exercises. J Bodyw Mov Ther 2014;18(1):34-41.

16. Comerford MJ, Mottram SL. Functional stability re-training: principles and strategies for managing mechanical dysfunction. Man Ther $2001 ; 6(1): 3-14$

17. Herrington L, Davies R. The influence of Pilates training on the ability to contract the Transversus abdominis muscle in asymptomatic individuals. Journal of Bodywork and Movement Therapies. 2005;9(1):52-57.

18. Miyamoto GC, Costa LOP, Galvanin T, et al. The efficacy of the addition of the Pilates method over a minimal intervention in the treatment of chronic nonspecific low back pain: a study protocol of a randomized controlled trial. J Chiropr Med. 2011;10(4):248-254.

19. Taylor SJ, Taylor AE, Foy MA, et al. Responsiveness of common outcome measures for patients with low back pain. Spine. 1999;24(17):1805-1812.

20. Finch E, Brooks D, Stratford PW, Mayo NE. Physical Rehabilitation Outcome Measures 2nd ed. Ontario, Canada, USA: BC Decker Inc; 2002.

21. Davidson M, Keating J. A comparison of five low back disability questionnaires: reliability and responsiveness. Phys Ther. 2002;82(1):824.

22. Kelly AM. The minimum clinically significant difference in visual analogue scale pain score does not differ with severity of pain. Emerg Med J. 2001;18(3):205-207.

23. Jensen MP, Chen C, Brugger AM. Interpretation of visual analog scale ratings and change scores: a reanalysis of two clinical trials of post operative pain. J Pain. 2003;4(7):407-414.

24. Gallagher EJ, Liebman M, Bijur PE. Prospective validation of clinically important changes in pain severity measured on a visual analog scale. Ann Emerg Med. 2001;38 (6):633-638.

25. Waldhelm A, Li L. Endurance tests are the most reliable core stability related measurements. Journal of Sport and Health Science. 2012;1(2):121-128.

26. Abdelraouf OR, Aziem AAA. The relationship between core endurance and back dysfunction in collegiate male athletes with and without nonspecific low back pain. Int J Sports Phys Ther. 2016;11(3):337-344.

27. Akaha H, Matsudaira K, Takeshita K, et al. Modified measurement of finger-floor distance: self-assessment bending scale. The Journal of Japanese Society of Lumber Spine Disorders. 2008;14(1):164-169.

28. Kamioka H, Tsutani K, Katsumata Y, et al. Effectiveness of Pilates exercise: A quality evalualtion and summary of systematic reviews based on randomized controlled trials. Complement Ther Med. 2016;25:1-19.
29. Natour J, Cazotti LDA, Ribeiro LH, et al. Pilates improves pain, function and quality of life in patients with chronic low back pain: A randomized controlled trial. Clin Rehabil. 2015;29(1):59-68.

30. Physical activity guidelines for adults (19-64 years). Gov UK; 2011. 1

31. Clinical Standards Advisory Group. Back Pain: report of CSAG Committee on Back Pain. London, UK: Her Majesty’s Stationary Office; 1994

32. Stolberg HO, Norman G, Trop I. Randomized controlled trials. AJR. 2004;183(6):1539-1544

33. Gill MSM, Childs A, Liebenson C. Endurance times for low back stabilisation exercises: clinical targets for testing and training from a normal database. Arch Phys Med Rehabil. 1999;80(8):941-944.

34. ACSM. Exercise management for persons with chronic diseases and disabilities. 3rd ed. Champaign, USA: IL Human Kinetics; 2009.

35. Taylor LA, Hay-Smith EJ, Dean S. Can clinical Pilates decrease pain and improve function in people complaining of non-specific chronic low back pain? A pilot study. New Zealand Journal of Physiotherapy. 2011;39(1):30-39.

36. Searle A, Spink M, Ho A, et al. Exercise interventions for the treatment of chronic low back pain: a systematic review and meta-analysis of randomised controlled trials. Clin Rehabil. 2015;29(12):1155-1167.

37. Rio E, Ark VM, Docking S, et al. Isometric contractions are more analgesic than isotonic contractions for patellar tendon pain: An in-season randomized clinical trial. Clin J Sport Med. 2016;27(3):253-259.

38. Slade SC, Keating JL. Trunk-strengthening exercises for chronic low back pain: a systematic review. J Manipulative Physiol Ther. 2006;29(2):163173.

39. Richmond J. Multi-factorial causative model for back pain management; relating causative factors and mechanisms to injury presentations ad designing time and cost effective treatment thereof. Med Hypotheses 2012;79(2):232-240

40. Kumar T, Kumar S, Nezamuddin D, et al. Efficacy of core muscle strengthening exercise in chronic low back pain patients. $J$ Back Musculoskelet Rehabil. 2015;28(4):699-707.

41. Vega MD, Marban MR, Viciana J. Criterion-related validity of sit-andreach tests for estimating hamstring and lumbar extensibility: a metaanalysis. J Sports Sci Med. 2014;13(1):1-14. 